\title{
Frank Seeliger*
}

\section{Bibliotheksinformatik studieren heißt: Was Hänschen nicht lernt, lernt Hans hinterher}

https://doi.org/10.1515/bfp-2019-2057

Zusammenfassung: Der Beitrag thematisiert die berufliche Fortbildung in Bezug auf den in Wildau seit 2015 angebotenen Masterstudiengang Bibliotheksinformatik und beschreibt, vor welchem Hintergrund man sich bei der Vielzahl an möglichen IT-Skills auf die zehn angebotenen Module fokussiert hat. Er reflektiert kritisch die bisher gewonnenen Erfahrungen aus Sicht der Konstrukteure des Curriculums, aber ebenfalls der Absolventen.

Schlüsselwörter: Informatik; Erfahrungsbericht; Fernstudium

\section{Studying Computer Science for Libraries Means: "You Can't Teach Old Dogs New Tricks” is History!}

Abstract: This paper presents a part-time master's programme in computer science for libraries (librarians?). This four-semester programme has been on offer at $\mathrm{TH}$ Wildau since 2015. In this paper the perspectives of academics and trainers and of students involved in the programme will be explored and assessed.

Keywords: Computer science; part-time programme; report of experiences

Auf diese Formel, was Hänschen nicht lernt, lernt Hans hinterher, zum Erwerb geistiger Kompetenzen aus kognitionspsychologischer Perspektive bei Erwachsenen bringt es die bekannte ETH-Bildungsforscherin Elsbeth Stern in einem Buchbeitrag. ${ }^{1}$ Automatische Routinen helfen, sich auf den Inhalt eines Textes zu konzentrieren, Vorwissen lässt schneller anknüpfen und das metakognitive Wissen kann Erwachsenen das Lernen ebenfalls erleichtern. Insofern lernen Kinder neu und Erwachsene dagegen knüpfen an oder lernen um, wie es im Untertitel dieses Beitrags von Stern heißt. Der Beitrag machte Mut und gab mit Anlass, in

1 Stern (2006).

*Kontaktperson: Dr. Frank Seeliger, fseeliger@th-wildau.de ein Projekt Zeit und Kraft zu investieren, um eine gefühlte Lücke zu füllen: die der fachbezogenen IT-Kompetenz.

Aber womit fängt man an, oder anders gefragt, in der schnelllebigen Zeit, „Wer kommt da noch mit?“. So lautet der sehr aufschlussreiche Beitrag von Schnabel et al. (2018) in DER ZEIT, u. a. mit der Quintessenz:

„Dass nichts beständiger sei als der Wandel, predigte schon vor 2500 Jahren der Philosoph Heraklit; doch heute kommen immer mehr Menschen nicht mehr mit. Deshalb verdiene sie ihr Geld damit, ,bei Vorträgen nicht die Zukunft zu erklären und noch nicht mal die Gegenwart, sondern Veränderungen, die schon seit fünf bis zehn Jahren eingetreten sind', erklärte einmal Kathrin Passig, preisgekrönte Schriftstellerin und Technik-Bloggerin. Denn viele der revolutionären Techniken, über die Berater und Forscher redeten, seien bereits da. ,Man hat die Nase schon vorn, wenn man es schafft, als Privatperson oder Firma nicht den Kontakt zur Gegenwart zu verlieren. ““

Und besser lassen sich die zehn themenspezifischen Module des berufsbegleitenden Masterstudienganges Bibliotheksinformatik in ihrer Intention nicht wiedergeben. Das seit 2015 in Wildau angebotene Studienprogramm ist nicht die Avantgarde beim Thema Forschungsdatenmanagement, Künstlicher Intelligenz, maschinelle Erschließung, Langzeitarchivierung o.ä. Auch folgt der modulare Aufbau des berufsbegleitenden Masterstudienganges Bibliotheksinformatik nicht dem roten Faden, dem das gerade erschienene Buch bei De Gruyter mit dem Titel Praxishandbuch IT-Grundlagen für Bibliothekare von Elke Lang und Andreas Bohne-Lang folgt. ${ }^{2}$ RFID und Makerspace sind im Studiengang kein Thema. Trotzdem beanspruchen beide berechtigterweise Grundlagen der Informatik zu vermitteln, die zum Verständnis der Anwendungen im bibliothekarischen Alltag benötigt werden. Vielleicht ist es an dieser Stelle einfach nur passend, aus der Festrede einer Absolventin, Tracy Hoffmann, vom 30. November 2018 zu zitieren. Darin es heißt:

\footnotetext{
"About ten years ago libraries complained about Google, by playing devil's advocate like: 'do we need libraries anymore, it's all inside the internet!' I am happy to see, that these times are over and that recently libraries became confident again. Some of them began developing their own digital infrastructure and also
}

2 Lang und Bohne-Lang (2019). 
became independent from companies, selling bad software to overcharged prices. This was the time when the demand of IT staff exploded rapidly. To hire IT experts is one way, but to talk about the specific needs and requirements of a library is another. So, there was (and probably there is) sometimes a huge or a small gap - filled with misinterpretation and misunderstanding - between the IT and the library world. And this is where the library informatics comes in. The graduates of these studies are supposed to fill the gap and support both sides. They will do requirements engineering, act as a mediator between library departments and the IT department, write programs, invent new services and overall - they will do nothing less than - developing the future of libraries. I'm confident that we - the graduates will meet these high expectations."

Basis des Curriculums sind die Grundlagen des Gegenwärtigen, wie sie über entsprechende Workshops, Vorträge und Projekte wahrgenommen wurden. Was den mittlerweile 43 Studierenden in den vier Jahrgängen aus 13 Bundesländern, zudem aus der Schweiz und Italien, in den vier Semestern bevorsteht, sind klassische Kurse zu Programmiersprachen, dem Internet als ein Service, Schnittstellen, Metadatenmanagement, IT-Sicherheit, Projektmanagement, Suchmaschinenalgorithmen, Datenbankmanagement usf. Der Studiengang mit seinen Modulen und Modulkatalogen $\mathrm{zu}$ Lernzielen und Inhalten, Abläufen, Rhythmen ist gut online dokumentiert, ebenfalls die bisherigen Beiträge über ihn verraten eine hohe Transparenz. ${ }^{3}$ Möchte man sich darüber informieren, findet man eine gute Entscheidungsgrundlage in den dort hinterlegten Dokumenten, medialen Aufbereitungen und Testimonials.

Eine wichtige Voraussetzung für die Aufnahme des Studiums ist die Berufserfahrung. Die Studierenden rekrutieren sich dabei aus Mitarbeitern von Hochschul-, Staats-, National- und Landesbibliotheken, Informations- und Forschungseinrichtungen, Wissenschaftlichen wie Öffentlichen Bibliotheken oder gar aus dem Archivbereich.

Zur sinnstiftenden Einordnung des Handelns sind die Kenntnisse der hiesigen Bibliothekslandschaft mit all ihren Facetten, aber auch über bisherige technische Grenzen unerlässlich. Dieser Landschaft an Netzwerken, Schnittstellen, Kooperationen und Projekten wird mit Blick auf interne wie externe Abläufe oder die Serviceorientierung und Erschließung neuer Märkte über die zunehmende $\mathrm{Au}$ tomatisierung, Digitalisierung und Technisierung ein digital turn eingehaucht. Und anders als in Curricula von Digital Humanities, Data Science, Data Curation hat der Studiengang Bibliotheksinformatik nach wie vor den verführerisch gesamten Betrieb einer Informationseinrich-

3 https://www.wit-wildau.de/studienprogramme/bibliotheksinfor matik. tung im Blick. Ausschließlich record und data management $\mathrm{zu}$ betreiben, ist nicht die tragende Idee dieser Ausbildungsinitiative.

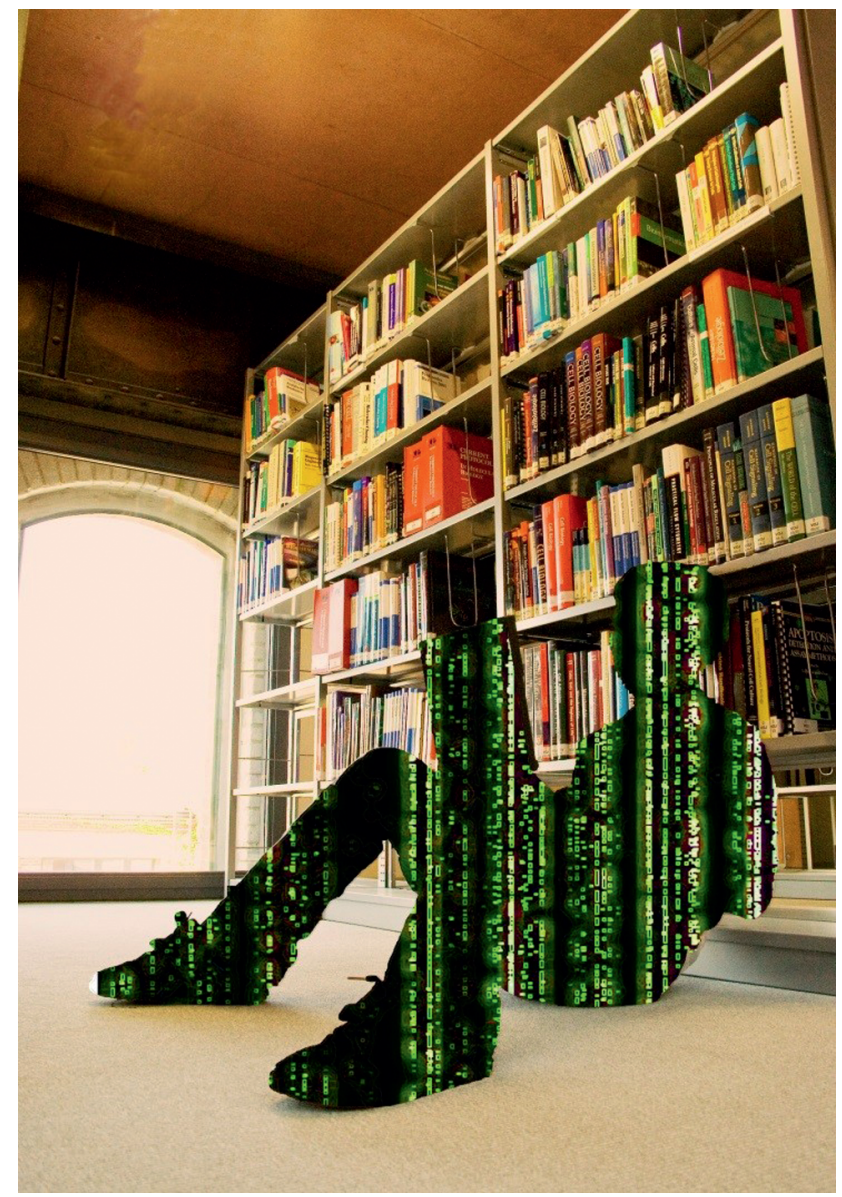

Abb. 1: Junktim zweier Welten (CFoto: Fernando Valdivieso und Vanessa Lierse)

Ob dieser holistische Ansatz den Studiengang auf Dauer tragen wird, muss sich zeigen, denn mit Blick auf die vier Matrikel muss man festhalten, dass das Interesse an der Ausbildung zwar ungebrochen ist, sich das Angebot und die Nachfrage knapp die Waage halten, insofern die Finanzierung um den Break-even schwangt. Starteten wir mit der Miller'schen Zahl an Teamgröße gemäß des Klassikers in der Psychologie The Magical Number Seven, Plus or Minus Two, ${ }^{4}$ wird mittlerweile die Zielgröße der zwölf Jünger erreicht. Im Resümee verlassen bislang aber auch in jedem Jahrgang zwei Studierende aus verschiedenen

4 Miller (1956). Neudeutsch im Slang von Amazons Jeff Bezos spricht man auch von der two-pizza rule, in dem Sinne, dass eine Teamgröße nicht umfänglicher sein darf, wie eine Pizza sie ernähren kann, ansonsten stellen sich der Teamgröße geschuldete inhärente Nachteile ein, vgl. Hern (2018). 
Gründen den Studiengang. Insofern werden die kommenden Jahre das anhaltende oder abnehmende Interesse an dem ohne Frage ressourcenverzehrenden Studiengang zeigen.

Die Ressourcen Zeit und Energie werden auf einer Durststrecke von mindestens zwei Jahren abverlangt, in denen vieles Weitere an wichtigen Punkten der Lebensqualität zurückgestellt werden muss, um den hohen Arbeitsanforderungen gerecht zu werden. Alle zwei Monate findet eine Präsenzwoche in Wildau statt, was sich auf insgesamt 450 Unterrichtseinheiten à 45 Minuten summiert. Und dies ist nur ein Drittel des notwendigen Workloads, der für gute Leistungen zu liefern ist, denn die anderen zwei Drittel werden zu Hause erbracht. Via Moodle und anderen Tools werden Aufgaben bearbeitet, Lehrstoff nach- und Präsentationen vorbereitet. Keine Studierenden, und die deutliche Mehrheit sind Kolleginnen, würde das Umschreiben mit „Malochen“ als befremdlich befinden. Die meisten Studierenden sind in Teilzeit beschäftigt, um das Arbeitspensum zu stemmen. Und bislang absolvierte niemand das Studium in der Regelstudienzeit von vier Semestern, sondern es musste mindestens ein fünftes herangezogen werden, um die Früchte alles eigenen Engagements einzufahren, wie das abschließende Schwingen der Quaste oder Tassel auf dem mortarboard von rechts nach links symbolisiert.

Die ersten Masterabschlüsse sind erfolgreich verliehen, es wird der Praxistest im Berufsfeld zeigen, ob die notwendigen Kompetenzen und Instrumente den Absolventen an die Hand gegeben werden konnten, um für Bibliotheken maßgeschneiderte IT-Produkte in die Geschäftsgänge $\mathrm{zu}$ integrieren. Mit anderen Worten beginnt damit erst der eigentliche Praxistest des apriorisch geformten Curriculums, auch wenn post festum schon erste Rückmeldungen wie von Uschi Klute (Verbundzentrale des GBV (VZG) und eine der ersten Absolventinnen der Bibliotheksinformatik) vorliegen, wo es heißt:

„Die im Studium erworbenen Kenntnisse und Fertigkeiten helfen mir an meinem Arbeitsplatz, IT-Probleme zu analysieren und Lösungen $\mathrm{zu}$ entwickeln. Durch vielfältige Unterrichtsinhalte und praktische (Programmier-)Aufgaben verfüge ich jetzt über Kompetenzen, um meine Schnittstellenfunktion zwischen Bibliotheken und Systemtechnik deutlich besser auszufüllen. Trotz des hohen Arbeitspensums habe ich das Studium als außerordentlich bereichernd empfunden.“

Unabhängig des Erreichten und der abzuwartenden repräsentativen Evaluierung in der Praxisnähe wird sich der Studiengang weiterentwickeln und den Erfordernissen anpassen. Drei Szenarien seien als möglicher Ausblick kurz angerissen: Das Modul Projektmanagement soll binnen dieses Jahres erweitert werden, um Komponenten wie Change Management und Leadership. Weiterhin gibt es aktive Überlegungen, ECTS-taugliche Module inner- und außerhalb eines Curriculums wie z. B. über Künstliche Intelligenz anzubieten, um damit berufliche und gleichzeitig anrechenbare Weiterbildung anzubieten, die teils trendnäher ausgerichtet sein kann.

Aufgeschlossen und kritisch beobachten wir eine Entwicklung, dass zunehmend berufsbegleitende Masterstudiengänge ohne Erststudium ermöglicht werden. Ein Pilotprojekt an der TH Brandenburg mit dem Studiengang Security Management wird aufmerksam verfolgt. ${ }^{5}$ Hierbei geht eine Eingangsprüfung voraus, die sich aus Prüfung der Kenntnisse auf Bachelorniveau und einem Brückensemester zusammensetzt.

Um mit einem Bonmot abzuschließen und meiner Generation für z.B. dieses Lebensexperiment noch einmal Mut zuzusprechen, sei es mir gestattet, Hirschhausen zu strapazieren, der festhält: „Die Jüngeren können schneller rennen, die Älteren kennen die Abkürzung.“6

\section{Literaturverzeichnis}

Stern, Elsbeth (2006): Was Hänschen nicht lernt, lernt Hans hinterher. In: Vom Lernen zum Lehren: Lern- und Lehrforschung für die Weiterbildung, hg. v. Ekkehard Nuissl. Bielefeld: Bertelsmann, 93-105.

Lang, Elke; Bohne-Lang, Andreas (2019): Praxishandbuch IT-Grundlagen für Bibliothekare. Berlin: De Gruyter.

Schnabel et al. (2018): Titel. DIE ZEIT, Nr. 52, 13.12.2018.

Miller, George A. (1956): The Magical Number Seven, Plus or Minus Two: Some Limits on Our Capacity for Processing Information. In: The Psychological Review, 63, 81-97, doi:10.1037/h0043158.

Hern, Alex (2018): The two-pizza rule and the secret of Amazon's success. In: The Guardian, 24. April 2018.

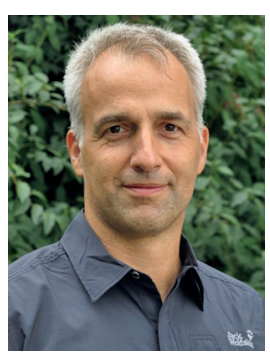

Dr. Frank Seeliger

Leiter der Hochschulbibliothek TH Wildau Hochschulring 1 D-15745 Wildau fseeliger@th-wildau.de

5 Vgl. zdd.th-brandenburg.de/studieren/master-ohne-bachelor. 6 Interview in DIE ZEIT vom 20. September 2018, Nr. 39/2018 bzw. online unter zeit.de/2018/39/bessere-haelfte-eckart-von-hirschhausen-tobias-esch-alt-werden/komplettansicht. 